\title{
Particle Physics in the Cosmos: Session Summary
}

\section{Basudeb Dasgupta}

Tata Institute of Fundamental Research, Homi Bhabha Road, Mumbai 400005, India

E-mail: bdasgupta@theory.tifr.res.in

\section{Karoline Schaeffner}

GSSI - Gran Sasso Science Institute, L'Aquila, 67100, Italy

E-mail: karoline.schaeffner@lngs.infn.it

This manuscript presents a summary of the parallel session V "Particles in the Cosmos" of the Neutrino Oscillation Workshop - NOW 2018. The topics covered by the session are a combination of new theoretical approaches and ideas and a selection of recent experimental results and upcoming projects.

Neutrino Oscillation Workshop (NOW2018)

9 - 16 September, 2018

Rosa Marina (Ostuni, Brindisi, Italy) 


\section{Introduction}

Session V was devoted to the role of "Particle Physics in the Cosmos." In the following we will give a brief summary of the contributions to this session of the NOW 2018 workshop. The topics cover, on the one hand, theories explaining the baryon asymmetry, physics beyond the Standard Model of Cosmology, and theoretical frameworks for new dark matter candidates, to on the other hand, a selection of latest results from WIMP and WIMP-like dark matter searches and a new experimental approach in the recently very active field of coherent elastic neutrino-nucleus scattering.

\section{Theorists' Perspective}

\subsection{Explaining the Baryon Asymmetry}

The generation of the dark matter relic density and the baryon asymmetry of the Universe are among the major puzzles of modern physics. The probably most popular solution for the second puzzle is represented by the leptogenesis mechanisms.

Giorgio Arcadi discussed that, among the different realizations, the Akhmedov-RubakovSmirnov mechanism ensures a successful baryogenesis via leptogenesis by extending the Standard Model with degenerate right-handed/sterile neutrino with mass scale not exceeding the GeV scale. This high mass degeneracy is related, in some scenarios for the generation of the SM neutrino masses, the linear and the inverse see-saw, to a tiny violation of the lepton number, being technically natural. The results of a thorough numerical analysis of these scenarios were presented, showing that successful leptogenesis occurs in regions of the parameter space which can be probed at present and future experimental facilities [1].

Stefano Morisi discussed an extension of the Standard Model with three heavy right-handed neutrinos. In the proposed mechanism, active neutrinos get a mass through the seesaw mechanism and baryon asymmetry is generated through leptogenesis. Moreover, they considered a reasonable hierarchical structure for the Dirac neutrino mass matrix, similar to the up-type quark mass matrix. Under such a conditions a relevant connection between the high scale CP violation and low energy Dirac neutrino CP phase emerges. Further, leptogenesis requires a compact heavy right-handed neutrino mass spectrum, which in turn implies a relation between low energy neutrino parameters. In particular, the Dirac CP phase is restricted in the range $-0.90 \pi<\delta<-0.75 \pi$ and the lightest active neutrino mass in the range $m_{1} \sim(0.002-0.004) \mathrm{eV}$ [2].

\subsection{Beyond the Standard Model of Cosmology?}

The $\Lambda$ CDM standard model of cosmology, a flat Universe dominated by a cosmological constant and cold dark matter, is found to be in excellent agreement with observations. However, this paradigm is mostly tested by observations at very large scales. On the scales of dwarf galaxies, the model faces problems that have even been dubbed as the small-scale crisis of $\Lambda \mathrm{CDM}$ cosmology. While it is possible, and probably even likely, that these tensions have mundane astrophysical or observational explanations, it is interesting to wonder if and how they can be addressed by new particle physics. A simple model that does the job introduces a new gauge interaction through an $\mathrm{MeV}$-scale mediator. This leads to dark matter self-interactions solving the cusp-core, too big to 
fail, and diversity problems. In addition, a new light particle species interacts with the dark matter by the new interaction, which keeps the dark matter in kinetic equilibrium long after its freeze-out from chemical equilibrium. As a result, structure formation is suppressed at small scales, which solves the missing satellites problem. Thus, a simple particle physics model is able to address all small-scale problems of $\Lambda \mathrm{CDM}$ cosmology [3, 4, 5].

Joern Kersten discussed a tempting possibility: identifying the new light particle with the sterile neutrino associated with the LSND and MiniBooNE anomaly [6]. As the new interaction also suppresses sterile neutrino production by oscillations in the early universe, there is hope that the cosmological neutrino mass bound can be avoided. However, the most recent studies conclude that this is not possible because of sterile neutrino production at later times. In this case, the new particles have to be generic dark fermions that mix at most very weakly with the Standard Model neutrinos.

Subhajit Ghosh discussed the effects of such non-standard neutrino interactions on primordial gravitational waves which source polarization anisotropies in the CMB as B-modes [7]. New interactions of neutrinos can stop them from free streaming, suppressing the growth of anisotropic stress responsible for damping GWs, and thus enhance PGW and CMB B-modes. In the specific realization, neutrinos were coupled with dark matter maintaining electroweak gauge invariance. In the parameter space of the model, the DM- $v$ scattering cross-section was either independent of neutrino temperature $\left(\sim T_{v}^{0}\right)$ or proportional to the square of neutrino temperature $\left(\sim T_{v}^{2}\right)$. Because of the new interaction, CMB B-modes at large multipoles $(\ell)$, which correspond to small scale modes of PGW, get enhanced compared to $\Lambda \mathrm{CDM}$. A primordial tensor spectrum with positive tensor spectral index $n_{T}>0$, i.e., a blue spectrum, also enhances the CMB-B modes at high $\ell$ compared to $\Lambda \mathrm{CDM}$ for which $n_{T} \sim 0$. Therefore DM- $v$ interaction can mimic blue primordial tensor spectrum which is a UV characteristic of PGW. However, the shapes of these two types of modification are different, therefore, high precision CMB B-mode experiments should be able to differentiate between these two scenarios. DM- $v$ interaction is also constrained from CMB scalar modes, but these bounds can be weakened significantly in multicomponent DM scenario where only a fraction of the total DM interacts with neutrinos. Therefore in a multicomponent model, DM- $v$ interaction can have a larger effect on CMB-B modes.

Luca Vecchi focussed on neutrino oscillations in dark backgrounds [8]. The dynamics that completes the Standard Model must be either very heavy or very weakly-coupled to us: in either case its effects decouple from experiments, thus explaining the absence of any direct evidence so far. It was argued that neutrino oscillation experiments are sensitive to very feeble interactions between the Standard Model and dark sectors, as long as these are very light. This offers a unique probe of light new physics sectors coupled to us via the neutrino portal.

Josef Pradler discussed the possibility that our Universe is filled with dark radiation, such as SM neutrinos or new physics states, that are sourced by the decay of dark matter with cosmologically long lifetime. For neutrino dark radiation he discussed the experimental detectability in underground rare event searches. For new physics dark radiation he highlighted the implications for $21 \mathrm{~cm}$ astronomy and offered an explanation of the EDGES result [9]. 


\subsection{Other Dark Matter Candidates}

Julian Heeck discussed the possibility of majorons as dark matter. Majorons are the Goldstone bosons of spontaneously broken lepton number and hence intimately connected to Majorana neutrino masses. Since all majoron couplings are heavily suppressed by the seesaw scale they are interesting candidates for long-lived dark matter. The signature decay into two mono-energetic neutrinos is potentially detectable with neutrino detectors for majoron masses above $\mathrm{MeV}$ and complementary to the loop-induced decays into visible particles [10]. The mass range between $\mathrm{keV}$ and $\mathrm{MeV}$ can only be probed indirectly with the majoron decay into two photons; $\mathrm{keV}$-scale majorons can be warm or cold dark matter depending on the underlying freeze-in mechanism.

Ranjan Laha proposed a new way to most efficiently search for axion-like particles. The discovery of high-energy astrophysical neutrinos motivate the presence of sources which can produce photons up to an energy of a few PeV. Gamma-ray photons at these energies are most efficiently absorbed and thus can be used to search for axion-like particles. Photon - ALP conversion happens near the source magnetic field, and these ALPs can back convert into high-energy gamma-rays in the Milky Way magnetic field. Using this technique, existing and upcoming gamma-ray detectors, like HAWC, CTA, and LHAASO can probe new parts of the parameter space. LHAASO can be sensitive to couplings above $10^{-11} \mathrm{GeV}^{-1}$ and masses up to $3 \mu \mathrm{eV}$ [11].

\section{Experimentalists' Perspective}

\subsection{The Search for WIMP and Light Dark Matter}

Still today the nature of dark matter remains an unsolved puzzle. It was back in the 1970s when astronomers started to build a model which, based on a variety of observations today provides overwhelming evidence for the existence of dark matter in our Universe at a unprecedented precision level. The observations include temperature anisotropies of the cosmic microwave background, baryonic acoustic oscillations, type 1a supernovae as well as gravitational lensing studies and rotation curves of galaxies . However, the Standard Model of particle physics does not provide a particle candidate that could explain these observations. Consequently, the existence of dark matter requires physics beyond the Standard Model, making this specific field in astroparticle physics very attractive and explaining the huge interest and effort taken in the particle physics community to resolve this long-standing mystery. The list of possible dark matter candidates is very long, covering a mass range from ultralight bosons (so-called fuzzy dark matter) to massive primordial black holes. The latter gained particular interest after the first observations of gravitational waves from the merger of black holes by the experiments LIGO and Virgo.

The most popular dark matter candidate through the past decades is the so-called WIMP (Weakly Interacting Massive Particle). Assuming WIMP particles thermally produced in the early Universe and weak-scale interactions interestingly leads to the correct relic dark matter density. This is often referred to as the WIMP miracle, as hitting the weak scale by chance seems highly unlikely. The WIMP miracle may be considered as the starting point of a global effort to directly detect interactions of dark matter particles in earth-bound detectors. Thereby, the community established a so-called standard scenario, where dark matter particles are expected to scatter elastically and coherently off atomic nuclei. Apart from direct searches dark matter particles can also be 
searched for at colliders (missing mass) and in indirect detection experiments (annihilation products).

Despite enormous efforts within the last decade that extremely pushed the sensitivity to unexampled high level, in particular of direct detection experiments (next generation experiments will hit the neutrino floor), no particle other than a Standard-Model-compatible Higgs boson has been convincingly detected at the weak scale. The absence of a positive detection of the most popular dark matter candidates like WIMP/WIMP-like particles or axions and sterile neutrinos started to create a feeling of doom in the WIMP dark matter community but we should keep in mind that the evening crowns the day.

The strategy for the future as Marco Selvi from the XENON1T collaboration explained is to push the sensitivity and fully exploit the potential of present and near-future experimental facilities to further increase the sensitivity to eventually reach the neutrino floor. The XENON project aims to detect WIMPs using a double-phase xenon time projection chamber, operated deep underground in the INFN Laboratori Nazionali del Gran Sasso. They presented the most recent results obtained with the current detector, XENON1T, with a fiducial mass of $1.3 \mathrm{t}$, a data taking campaign of 279 days, corresponding to a $1.0 \mathrm{t} \cdot \mathrm{yr}$ exposure. The experiment obtained a record for their ultra-low background and set the strongest upper limit on the WIMP-nucleon cross section for WIMP mass larger than $6 \mathrm{GeV} / \mathrm{c}^{2}$ [12]. The next phase of the experiment, XENONnT, is already in preparation for 2019. They aim for an increase in target mass by a factor three and a reduced background, to achieve an improvement in sensitivity by a factor 10 in five years.

Liquid nobel gas searches like XENON1T, LUX, PandaX, DarkSide and their successors (e.g. XENONnt, LZ, DarkSide-20k) are the leading technology in the field of classical WIMP searches above about $10 \mathrm{GeV} / \mathrm{c}^{2}$. However, given that with next generation of such experiments most of the parameter space for the classical WIMP will be ruled out recently also low-mass dark matter below the WIMP scale started to be of interest in the community. In the context of light dark matter asymmetric dark matter models are considered a fruitful option as they, apart from a solution to the dark matter problem, also connect its generation to the baryon asymmetry. This reflects the idea beyond the WIMP-age of trying to expand the search in different directions and making use of all available technologies and facilities.

As reported by Luca Pattavina cryogenic detectors are a cutting edge technology in particular for the investigations of light dark matter thanks to their low energy thresholds. The CRESST (Cryogenic Rare Event Search with Superconducting Thermometers) experiment explores with high sensitivity the parameter space of low mass dark matter candidates, being the pathfinder in the sub-GeV/c ${ }^{2}$ mass range. CRESST-III employs scintillating $\mathrm{CaWO}_{4}$ crystals as target material operated at $\mathrm{mK}$ temperature. Thanks to the simultaneous read-out of heat, in the main absorber, and light, with a separate cryogenic light detector, the background in the region of interest is strongly suppressed. In 2018, the first stage of CRESST-III data taking was successfully completed, achieving an unprecedented energy threshold for nuclear recoils with their best detector (detector A, $\mathrm{CaWO}_{4}, 24 \mathrm{~g}$ ) of $26 \mathrm{eV}$ and thus setting a new benchmark in this field which is expected to open new frontiers in the near future [13]. Furthermore Luca Pattavina reported on an excess of events which they observe in their CRESST-III detectors in the low energy regime close to threshold. A new experimental run with improved and modified detectors already started and is subject to further clarifications on this observation. 
An alternative to cryogenic detectors but with sensitivity for low mass dark matter is NEWSG (New Experiments With Spheres-Gas), an experiment using Spherical Proportional Counters (SPCs) [14]. NEWS-G uses light noble gases to search for dark matter in the sub-GeV/c ${ }^{2}$ mass region. The NEWS-G project builds on the experience gathered with the so-called SEDINE detector, a $60 \mathrm{~cm}$ SPC which has been operating for several years at the Laboratoire Souterrain de Modane. The goal is to build now a $140 \mathrm{~cm}$ diameter SPC using even higher purity materials, and operate it at SNOLAB. Phillippe Gros reported the excellent results obtained with the SEDINE prototype with $\mathrm{Ne}$ as target nuclei, which exclude at $90 \%$ confidence level (C.L.) cross-sections above $4.4 \cdot 10^{-37} \mathrm{~cm}^{2}$ for a $0.5 \mathrm{GeV} / \mathrm{c}^{2}$ dark matter particle. An upgraded version is presently under construction with a deployment at SNOLAB planned for spring 2019.

The majority of experiments in the field of direct detection do not find a hint for the detection of dark matter yet. Remarkably though, the DAMA/LIBRA experiments observes since about 20 years an annual modulation signal which matches the expected signal from dark matter particles in the galatic halo, but is inconsistent with null results of numerous other direct dark matter searches. Since different experiments use diverse target materials, a direct comparison of their results is only possible if taking into account certain assumptions, in particular on the interaction mechanism between dark matter and ordinary matter. The only way of providing a fully model-independent clarification of the DAMA/LIBRA claim is the use of the same target material, but in a different experiment.

This is exactly what the COSINUS project (Cryogenic Observatory for SIgnals seen in Nextgeneration Underground Searches) aims for by employing in their experiment crystals of sodium iodide as target material, just as DAMA/LIBRA. Yet, different from other experiments that are currently under way to test the DAMA/LIBRA signal (COSINE-100, ANAIS, SABRE, Pico-lon) COSINUS will not operate the NaI crystals as pure scintillation detectors which solely acquire the scintillation light signal created by a particle interaction. Instead, COSINUS makes use of a novel detection approach based on the CRESST technology: NaI crystals operated as low-temperature scintillating detectors at milli-Kelvin temperatures measure two independent signals from a particle interaction, the heat signal and the simultaneously emitted scintillation light. This dual channel detection has two distinctive advantages explained Natalia di Marco: a lower energy threshold for nuclear recoils and background suppression by particle identification, a completely new feature for NaI-based searches. While the first generation of the cryogenic NaI prototype detectors arrive at an energy threshold of about $6 \mathrm{keV}$ thus ask for still some improvements to achieve the experimental goal of $1 \mathrm{keV}$, the sensitivity of the light detectors already meets the requirements [15]. The project recently received funding to move from an R\&D to a physics experiment. Already within its first phase, COSINUS- $1 \pi$ and a comparatively little exposure of $\mathrm{O}(100 \mathrm{~kg} \cdot \mathrm{d})$ they will be able to give insight whether DAMA sees a nuclear recoil signal, or not.

\subsection{Coherent Elastic Neutrino-Nucleus Scattering}

Coherent Elastic Neutrino-Nucleus Scattering (CEvNS) was predicted for the first time almost 40 years ago and in 2017 finally discovered by the COHERENT collaboration. Since then CEvNS become a very active and dynamic field of interest since it opens up new opportunities to probe physics beyond the Standard Model such as the search for a neutrino magnetic moment or sterile neutrinos. Johannes Rothe presented a novel cryogenic neutrino experiment with the name 
NU-CLEUS (ERC grant by R. Strauss) which will be operated at moderate distance to a nuclear power reactor and will allow for precision measurements of CE $v$ NS with miniaturized gram-scale detectors. The detector concept relies on cryogenic calorimeters based on the CRESST technology. With a recent NU-CLEUS demonstrator operated above ground using sapphire crystals as target they achieved an ultra-low threshold of $19.7 \mathrm{eV} \mathrm{[16].} \mathrm{For} \mathrm{the} \mathrm{full-optimized} \mathrm{version} \mathrm{they} \mathrm{report}$ that an energy threshold of below $10 \mathrm{eV}$ is feasible. For the first step NU-CLEUS-10g, operating a target array of $10 \mathrm{~g}$ only, they aim for a $\mathrm{O}(10 \%)$ measurement of the CEvNS cross-section within one year of data taking. This should, as explained by Johannes Rothe, already allow to explore new physics at the low-energy neutrino frontier which so far was not accessible by other experiments.

\section{References}

[1] A. Abada, G. Arcadi, V. Domcke and M. Lucente, "Neutrino masses, leptogenesis and dark matter from small lepton number violation?," JCAP 1712 (2017) no.12, 024

[2] F. Buccella, D. F. G. Fiorillo, G. Miele, S. Morisi, O. Pisanti and P. Santorelli, "Neutrino phenomenology from leptogenesis,” Eur. Phys. J. C 78 (2018) no.10, 817

[3] L. G. van den Aarssen, T. Bringmann and C. Pfrommer, "Is dark matter with long-range interactions a solution to all small-scale problems of $\Lambda$ CDM cosmology?," Phys. Rev. Lett. 109 (2012) 231301

[4] B. Dasgupta and J. Kopp, "Cosmologically Safe eV-Scale Sterile Neutrinos and Improved Dark Matter Structure," Phys. Rev. Lett. 112 (2014) no.3, 031803

[5] X. Chu and B. Dasgupta, "Dark Radiation Alleviates Problems with Dark Matter Halos," Phys. Rev. Lett. 113 (2014) no.16, 161301

[6] T. Bringmann, J. Hasenkamp and J. Kersten, "Tight bonds between sterile neutrinos and dark matter," JCAP 1407 (2014) 042

[7] S. Ghosh, R. Khatri and T. S. Roy, "Dark neutrino interactions make gravitational waves blue," Phys. Rev. D 97 (2018) no.6, 063529

[8] F. Capozzi, I. M. Shoemaker and L. Vecchi, "Neutrino Oscillations in Dark Backgrounds," JCAP 1807 (2018) no.07, 004

[9] M. Pospelov, J. Pradler, J. T. Ruderman and A. Urbano, "Room for New Physics in the Rayleigh-Jeans Tail of the Cosmic Microwave Background," Phys. Rev. Lett. 121 (2018) no.3, 031103

[10] C. Garcia-Cely and J. Heeck, "Neutrino Lines from Majoron Dark Matter," JHEP 1705 (2017) 102

[11] H. Vogel, R. Laha and M. Meyer, "Diffuse axion-like particle searches," arXiv:1712.01839 [hep-ph]

[12] E. Aprile et al., First Dark Matter Search Results from the XENONIT Experiment, Phys. Rev. Lett. 119 (2017) 181301

[13] F. Petricca et al., First results on low-mass dark matter from the CRESST-III experiment, arXiv:1711.07692 [astro-ph.CO]

[14] Q. Arnaud et al., First results from the NEWS-G direct dark matter search experiment at the LSM, Astropart. Phys. 97 (2018) 54-62

[15] K. Schäffner et al., "A NaI-Based Cryogenic Scintillating Calorimeter: Results from a COSINUS Prototype Detector," J. Low. Temp. Phys. 193 (2018) no.5-6, 1174.

[16] R. Strauss et al., Gram-scale cryogenic calorimeters for rare-event searches, Phys. Rev. D 96 (2017) 022009 